\title{
Extracorporeal Shock Wave Therapy Versus Phonophoresis Therapy for Neck Myofascial Pain Syndrome: A Randomized Clinical Trial
}

\author{
Parisa Taheri ${ }^{1}$, Marzie Naderi (iD ${ }^{1,{ }^{*}}$ and Saeid Khosravi ${ }^{1}$ \\ ${ }^{1}$ Department of Physical Medicine and Rehabilitation, School of Medicine, Isfahan University of Medical Sciences, Isfahan, Iran \\ "Corresponding author: Department of Physical Medicine and Rehabilitation, School of Medicine, Isfahan University of Medical Sciences, Isfahan, Iran. Email: \\ mn100000@gmail.com
}

Received 2020 December 29; Revised 2021 March 06; Accepted 2021 March 12.

\begin{abstract}
Objectives: This study aimed to assess the effects of using Extracorporeal Shock Wave Therapy (ECSWT) and phonophoresis therapy on pain and neck disability in patients with neck myofascial pain syndrome (MPS).

Methods: Forty eligible patients were randomly divided into two groups of ECSWT(received three sessions of ECSWT, once a week for three weeks) and phonophoresis (received ultrasound using hydrocortisone gel 1\% over the trigger point on trapezius muscle, three times a week for three weeks). Patients in both groups received the same stretching exercise program and drug regimen during the intervention.

Results: Pain and NDI scores in both groups were significantly improved at the end of the treatment and four weeks later. At the end of the treatment, the pain score was similar between the groups. Four weeks after the treatment, the pain score in the ECSWT group was significantly lower than in the phonophoresis group (P-value $=0.030)$. The NDI score was not significantly different between the groups at the end of the treatment. However, four weeks after the treatment, the NDI score was significantly lower in the ECSWT group than in the phonophoresis group (P-value=0.032). The trend of changes in the pain and NDI scores was not significantly different between the groups.

Conclusions: Both phonophoresis and ECSWT groups effectively decreased pain and neck disability in patients with MPS, with the superiority of ECSWT with a more lasting effect for a month after the end of the treatment.
\end{abstract}

Keywords: Myofascial Pain Syndrome, Extracorporeal Shock Wave Therapy, Phonophoresis, Neck Disability

\section{Background}

Neck pain occurs commonly throughout the world and is one of the leading causes of disability, which is associated with substantial disability and economic cost (1). The yearly prevalence of neck pain in the general population has been reported between 16.7 and $75.1 \%$. (1-3) The global point prevalence of neck pain was $4.9 \%$ (4), and in the global burden of disease 2010 study, neck pain had the fourth rank of disability among all 291 studied conditions (5). Absence from work due to a medical condition was due to a musculoskeletal disorder in $75 \%$ of the individuals (6). Thus, neck pain, as one of the leading causes of work absenteeism and reduced work productivity, increases healthcare costs on individuals and healthcare systems $(7,8)$.

The role of myofascial trigger points in pain processes in patients with mechanical neck pain has been proposed during the past decade. There are muscular hard bands in trigger points, and the pain returns ambiguously or in- tensely with different severities (9). Two studies have been conducted on myofascial pain syndrome (MPS) among patients suffering from neck pain $(9,10)$, and in a recent population-based study, all participants with chronic nonspecific neck pain had MPS (10).

MPS is a well-known regional pain condition of skeletal muscle fibers characterized by myofascial trigger points, with an estimated overall prevalence of about $46 \%$ (11). As one of the leading causes of disability in musculoskeletal system problems, MPS is more prevalent at the age of 30 to 60 and in women than men $(12,13)$. Since the exact pathophysiology and etiology of MPS are still unknown, the treatment approaches mostly target symptoms. Pharmacotherapy, injection therapy, physical therapy, and behavioral modification are traditional therapeutic approaches to MPS treatment (14).

In recent years, extracorporeal shock wave therapy(ECSWT) and ultrasound as non-invasive methods have expanded to MPS treatment. Studies have shown some de- 
gree of efficacy to apply ECSWT in MPS treatment. One study demonstrated the efficacy of high-energy ECSWT on alleviating pain in patients (15). Other studies show the same efficacy of low-energy ECSWT compared to a placebo (16). A randomized pilot study showed that both highand low-energy ECSWT effectively improved MPS symptoms with the superiority of high-energy ECSWT (17). On the other hand, regarding applying ultrasound in MPS treatment, some clinical trials established the benefits of ultrasound, while other studies did not show any significant effect of ultrasound (15).

As a non-invasive, painless method, phonophoresis is the use of ultrasound to increase skin absorption and penetration of topically applied drugs to deep tissues (18). For patients with musculoskeletal conditions, phonophoresis is used with topical anti-inflammatory drugs to reduce pain and inflammation (19). In MPS treatment, phonophoresis is reported to be more effective than conventional ultrasound (20-23). However, no study investigated the effect of phonophoresis in contrast to ECSWT on MPS treatment.

\section{Objectives}

This study aimed to compare the efficacy of phonophoresis with hydrocortisone gel and ECSWT on pain and physical disability in patients with neck MPS.

\section{Methods}

After the study protocol was approved at the Institutional Review Board and Ethics Committee of the Isfahan University of Medical Sciences and registration of the study for public registry code in Iran (IRCT 20190618043931N2), 40 eligible patients with myofascial pain of neck from physical medicine clinics in Isfahan, Iran, were enrolled. Patients older than 18 years old were included in the study if they had pain in at least one active trigger point located in the upper trapezius muscle for at least six months. The exclusion criteria were treatment programs for neck or shoulder pain during the last two months, neck or shoulder surgery during the last two years, neck radiculopathy, trauma history, cardiovascular, respiratory, or allergic disease, neck osteoarthritis, coagulation disorders, and pregnancy.

After informing the patients about the study objectives and obtaining signed written informed consent, they were randomly assigned into two groups. Randomization was allocated by Random Allocation software. Twenty patients were assigned to the ECSWT group, and 20 were assigned to the phonophoresis group. Patients in both groups received the same stretching exercise program for upper trapezius muscle, and the drug regimen included tizanidine at bedtime and meloxicam (7.5 mg/day) for three weeks.

The ultrasound was done in the phonophoresis group by applying hydrocortisone gel $1 \%$ (circularly with a thickness of $2-3 \mathrm{~mm}$ ) with $1 \mathrm{MHz}$ frequency and $1.2 \mathrm{Wt} / \mathrm{cm}^{2}$ power over the trigger points on the trapezius muscle for 10 minutes. In this group, therapies were applied three times a week for three weeks.

Patients in the ECSWT group received three sessions of ECSWT with radial prob once a week for three weeks. ECSWT was applied using an electromagnetic type Dornier AR2 machine (the standard electromagnetic DUOLITHSD1, Storz Medical, Tagerwilen, Switzerland) adjusted to the following settings: 2,000 pulses were applied at each session at an intensity of $0.2 \mathrm{~mJ} / \mathrm{mm}^{2}$ with $10 \mathrm{~Hz}$ frequency.

The treatments were performed in the same environmental condition by the same therapist. For data collection, the same physician evaluated all patients before, immediately after, and four weeks after the treatment.

This study's primary outcomes were pain intensity and the degree of physical disability measured before, immediately after, and four weeks after the treatment. Pain intensity was assessed by the visual analog scale (VAS, 0 - 10 $\mathrm{cm}$; 0 means no pain, 10 means the worst possible pain). The degree of physical disability was measured using the Neck Disability Index (NDI). The NDI is a multidimensional and self-administered index containing 10 items related to functional activity, symptom, and concentration. After adding all scores from the questions, the final NDI score was calculated for the patients. A higher score indicated increased functional disability related to cervical abnormality.

After data collection, SPSS version 25 (SPSS, Inc., Chicago, IL, USA) was used for final analyses. Mean \pm standard deviation (SD) or number (\%) were used for descriptive data, as appropriated. Before data analysis, the normality of the data was assessed by the Shapiro-Wilk test. The pain and NDI scores in each time point were compared between the groups using an independent sample t-test. ANCOVA was used for variables between the groups at the end of the treatment and four weeks after the treatment by controlling baseline values as a covariate. Also, the trend of the pain and NDI scores was compared within and between the groups using repeated 
measurements of ANOVA. The level of significance was considered to be less than 0.05 .

\section{Results}

To select eligible patients, 52 patients were examined, of whom nine were not eligible, and three refused to sign informed consent. The baseline characteristics of patients in the groups are mentioned in Table 1. During the followup, one patient in the ECSWT group and two patients in the phonophoresis group were lost. In the final analyses, 19 patients in the ECSWT group and 18 patients in the phonophoresis group were included (Figure 1). The demographic data of the patients are listed in Table 1. Duration of pain was similar in patients in both ECSWT and phonophoresis groups $(\mathrm{P}$-value $=0.851$ )

Table 2 shows the comparison of pain and NDI between the studied groups. The pain score at baseline and end of the treatment was similar between ECSWT and phonophoresis groups (P-value $>0.05$ ). Four weeks after the treatment, the phonophoresis group's reported pain score was significantly higher compared to the ECSWT group (P-value $=0.026)$. This difference between the groups was statistically significant after controlling baseline values as covariate $(\mathrm{P}$-value $=0.030)$. The decrease in pain during the study period was statistically significant within each group (P-value $=0.0001)$, whereas the trend between the groups was not significantly different(P-value $=0.066$, Figure 2 ). The NDI score was not significantly different between the groups at baseline and end of the treatment (P-value > 0.05). Four weeks after the treatment, the NDI score was significantly lower in the ECSWT group than in the phonophoresis group (P-value $=0.032$ ). The difference between the groups was statistically significant after controlling baseline values as covariate $(P$-value $=0.030)$. The trend of NDI score changes was statistically significant within the groups (P-value $=0.0001$ ), however, not significant between the groups (P-value $=0.517$, Figure 3 ).

\section{Discussion}

This study focused on comparing the effects of phonophoresis with hydrocortisone gel and ECSWT on pain and physical disability in patients with neck MPS. Our results indicated that both treatment methods significantly improved pain intensity and neck disability immediately after and four weeks after the intervention. Although no significant difference was observed between ECSWT and phonophoresis at the end of the intervention, pain intensity and neck disability were significantly more reduced than phonophoresis in the ECSWT group four weeks after the end of the treatment, showing the prolonged effect of ECSWT compared to phonophoresis in MPS treatment.

The use of ECSWT in MPS treatment compared to placebo or other treatments has been reported in various studies $(24,25)$; however, there is limited evidence that focused ECSWT is useful for short-term neck pain in MPS (26). Müller-Ehrenberg showed the positive effect of ECSWT on pain relief in the treatment of MPS (16). In Roman et al.'s study, ECSWT was a significant improvement in pain intensity (27). Moreover, in a randomized pilot study by Park et al., two different regimens of ECSWT were compared, and they showed that both regimens were useful in reducing pain and physical disability in patients with MPS; however, high-energy was more effective (15). Similar to these findings, our results indicated that three ECSWT treatment sessions were practical in treating MPS and decreasing pain and physical disability in these patients. Although most studies reported positive effects of ECSWT on trigger points, the dose-dependent nature of ECSWT and the lack of a standard treatment protocol are the main disadvantages of this method.

On the other hand, the use of phonophoresis for MPS is limited to some studies. Ay et al. (22) investigated the effectiveness of both diclofenac phonophoresis and ultrasound therapy in pain reduction disability with the same effect in treating patients with MPS. Ustun et al. (23) performed a study to compare a eutectic mixture of local anesthetics cream phonophoresis with conventional ultrasound therapy and demonstrated that phonophoresis was more effective than conventional ultrasound therapy in terms of pain and neck disability in patients with MPS. Sarrafzadeh et al. (24) compared the effects of phonophoresis of hydrocortisone $1 \%$ and ultrasonic therapy in patients with an upper trapezius latent myofascial trigger point and detected a significant effect of phonophoresis in pain reduction with its superiority to ultrasonic therapy. Similar to these studies, our results showed the significant effect of phonophoresis of hydrocortisone $1 \%$ in pain relief and neck disability in patients with MPS. Myofascial pain reduction in ultrasound therapy is through its mechanical and thermal effects like providing deep tissue heating, increasing microcirculation, enhancing vascular and cell membrane permeability, and improving angiogenesis. Adding phonophoresis gel by increasing skin absorption causes deeper tissues by ultrasound and, consequently, more relaxation and pain relief. 

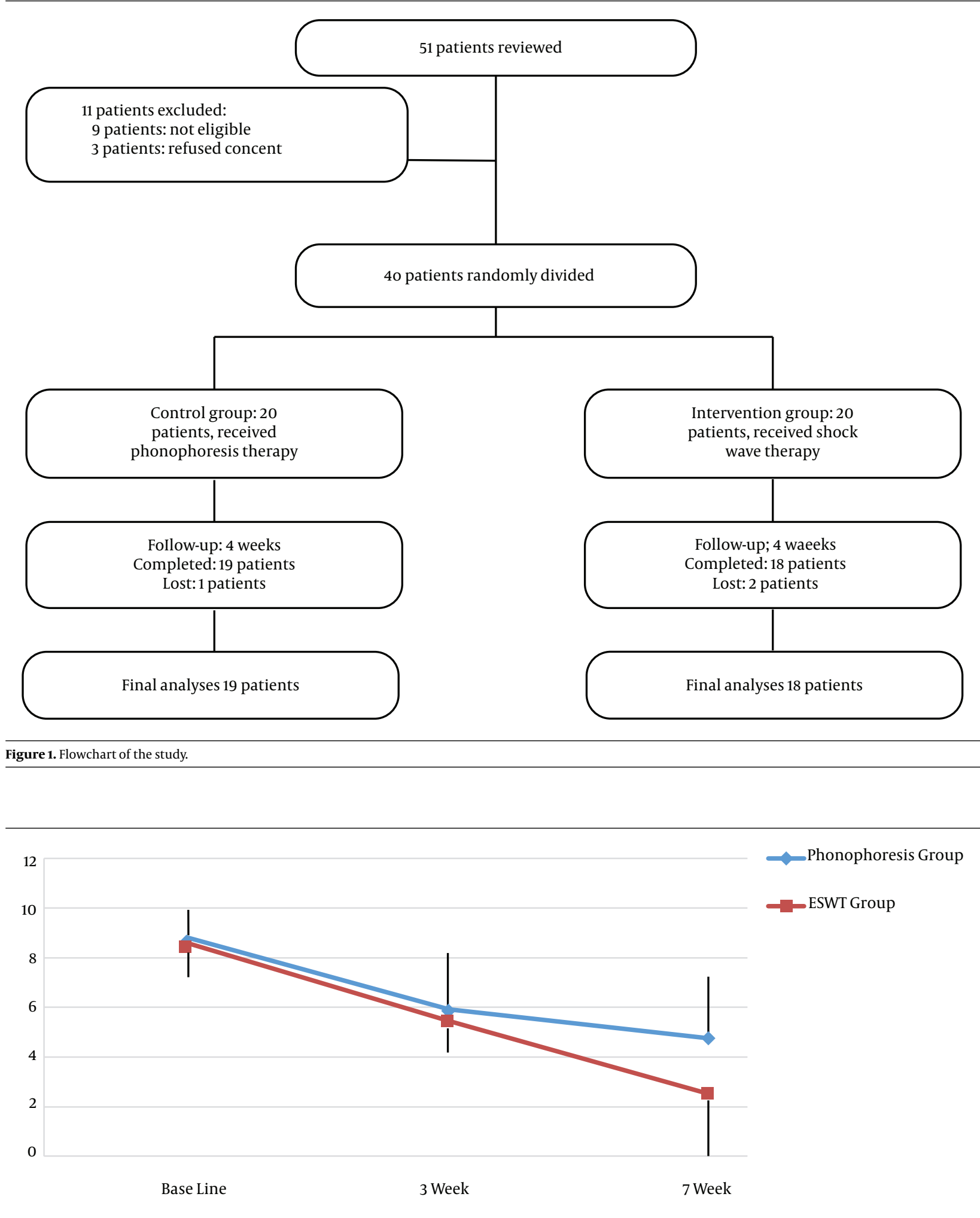

Figure 2. Trend of pain score during the study period between groups by repeated measurement of ANOVA $(\mathrm{P}$-values $=0.066)$. 


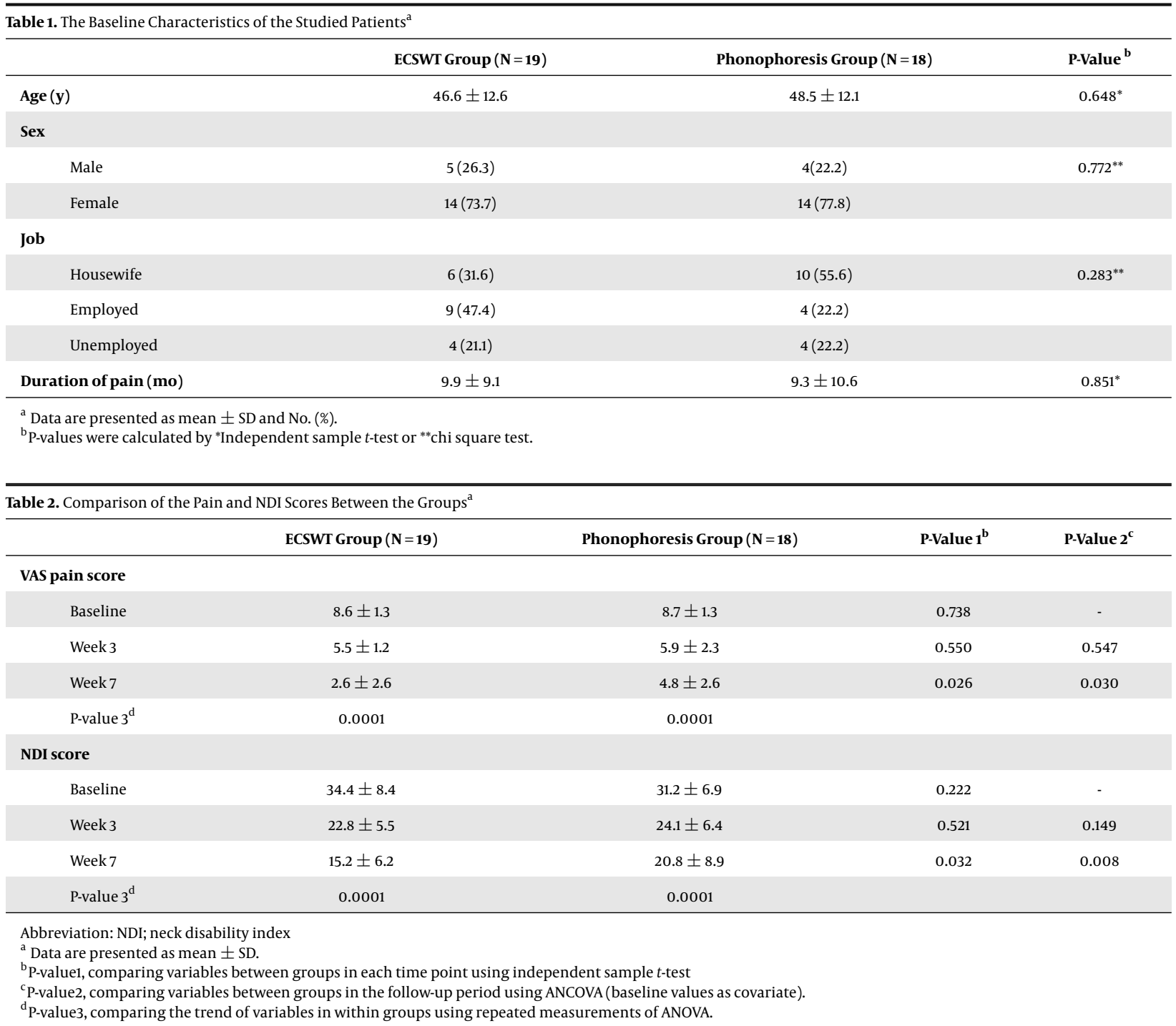

Based on our results, more significant improvement was observed on neck disability in the ECSWT group than in the phonophoresis group, four weeks after the end of the treatment. Lower neck disability in the ECSWT group can be related to functional improvement that can be explained by more pain relief of the upper trapezius in these patients. These results are comparable with Haghighat et al.'s study results, showing the favorable outcomes after four weeks of treatment with ESWT (28). However, our results are consistent with the results of a former study by Toghtamesh and colleagues, who found that one session of shock wave therapy in patients with MPS of trapezius muscle significantly decreased the VAS level and increased ROMs of lateral neck flexion (29).

To the best of our knowledge, no studies have investi- gated the comparison of ECSWT and phonophoresis in MPS patients. In a study encompassing 60 patients, Aktürk et al. compared the effectiveness of ECSWT and ultrasound therapy in MPS (30). They used four sessions with three-day intervals of ECSWT and 10 sessions of ultrasound therapy. They showed a significant effect of both ECSWT and ultrasound therapy in pain reduction compared to the control group, but they did not observe any significant difference between the two studied treatments. In contrast to Aktürk et al.'s findings, in our study, ECSWT had superiority to ultrasound therapy, even with the addition of phonophoresis with hydrocortisone gel $1 \%$ to ultrasound. The discrepancy between findings can be explained by different treatment protocols used in these two studies. In contrast to Aktürk et al., we used three sessions of ECSWT with one-week 


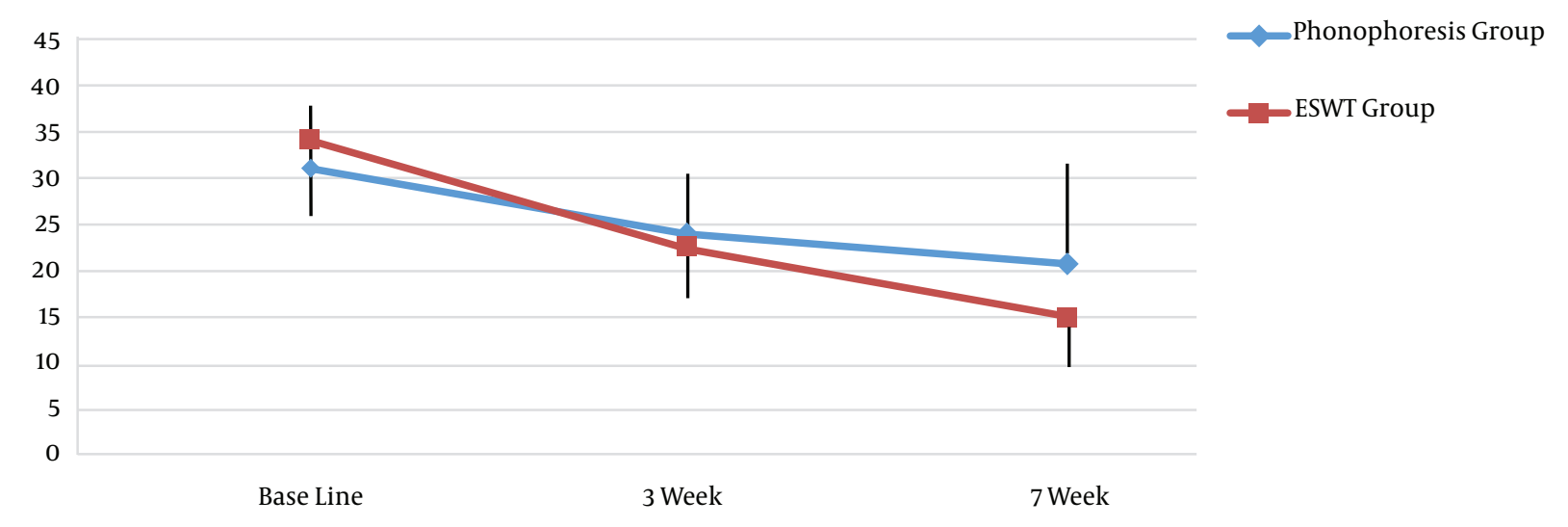

Figure 3. Trend of neck disability index score during the study period between groups by repeated measurements of ANOVA (P-values $=0.517$ ).

intervals, and in the phonophoresis group, we used nine sessions of phonophoresis.

This study had some limitations. First, the trained stretching exercise program was home-based, and during the follow-up period, the patients were not questioned about the duration of the exercises and compliance with this program. Second, we did not have control groups for the studied treatments, sham ECSWT, and conventional ultrasound therapy, which could be useful in clarifying the exact effect of adding the studied treatments to the stretching exercise programs and drug regimens. Thus, further studies may elucidate the effects of ECSWT and phonophoresis in MPS treatment.

\subsection{Conclusions}

The findings of the present study indicate that both phonophoresis and ECSWT treatments may effectively decrease pain and neck disability in patients with MPS. With a more lasting effect, one month after the end of the treatments, ECSWT show superior pain relief and neck disability results.

\section{Footnotes}

Authors' Contribution: Study concept and design: P.T. and M.N.; Acquisition of data: M.N.; Analysis and interpretation of data: S.K.; Drafting of the manuscript: M.N.; Critical revision of the manuscript for important intellectual content: S.K.; Statistical analysis: S.K.; Administrative, technical, and material support: P.T. and S.K.; Study supervision: P.T.

Clinical Trial Registration Code: Registration of the study for public registry code in Iran (IRCT 20190618043931N2).
Conflict of Interests: There is no conflict of interest.

Ethical Approval: The study protocol was approved by the Institutional Review Board and Ethics Committee of the Isfahan University of Medical Sciences (IR.MUI.MED.REC.1397.094).

Funding/Support: No funding or support was received for this study.

Informed Consent: Signed written informed consent was obtained from patients before the study.

\section{References}

1. Hogg-Johnson S, van der Velde G, Carroll LJ, Holm LW, Cassidy JD, Guzman J, et al. The burden and determinants of neck pain in the general population: results of the Bone and Joint Decade 2000-2010 Task Force on Neck Pain and Its Associated Disorders. J Manipulative Physiol Ther. 2009;32(2 Suppl):S46-60. doi: 10.1016/j.jmpt.2008.11.010. [PubMed: 19251074].

2. Shariat A, Tamrin SBM, Arumugam M, Danaee M, Ramasamy R. Prevalence rate of musculoskeletal discomforts based on severity level among office workers. Acta Medica Bulgarica. 2016;43(1):54-63. doi: 10.1515/amb-2016-0007.

3. Fejer R, Kyvik KO, Hartvigsen J. The prevalence of neck pain in the world population: a systematic critical review of the literature. Eur Spine J. 2006;15(6):834-48. doi: 10.1007/s00586-004-0864-4. [PubMed: 15999284]. [PubMed Central: PMC3489448].

4. Hoy D, March L, Woolf A, Blyth F, Brooks P, Smith E, et al. The global burden of neck pain: estimates from the global burden of disease 2010 study. Ann Rheum Dis. 2014;73(7):1309-15. doi: 10.1136/annrheumdis-2013-204431. [PubMed: 24482302].

5. Murray CJ, Vos T, Lozano R, Naghavi M, Flaxman AD, Michaud C, et al. Disability-adjusted life years (DALYs) for 291 diseases and injuries in 21 regions, 1990-2010: a systematic analysis for the Global Burden of Disease Study 2010. Lancet. 2012;380(9859):2197-223. doi: 10.1016/S01406736(12)61689-4. [PubMed: 23245608].

6. Malik KM, Beckerly R, Imani F. Musculoskeletal disorders a universal source of pain and disability misunderstood and mismanaged: A critical analysis based on the U.S. Model of care. Anesth Pain 
Med. 2018;8(6). e85532. doi: 10.5812/aapm.85532. [PubMed: 30775292]. [PubMed Central: PMC6348332].

7. Haldeman S, Carroll L, Cassidy JD. Findings from the bone and joint decade 2000 to 2010 task force on neck pain and its associated disorders. J Occup Environ Med. 2010;52(4):424-7. doi: 10.1097/JOM.0b013e3181d44f3b. [PubMed: 20357682].

8. Guzman J, Hurwitz EL, Carroll LJ, Haldeman S, Côté P, Carragee EJ, et al. A new conceptual model of neck pain. Eur Spine J. 2008;17(S1):14-23. doi: 10.1007/s00586-008-0621-1.

9. Ghasemi M, Mosaffa F, Hoseini B, Behnaz F. Comparison of the effect of bicarbonate, hyaluronidase, and lidocaine injection on myofascial pain syndrome.Anesth Pain Med.2020;10(3).doi: 10.5812/aapm.101037.

10. Fernandez-de-Las-Penas C, Cuadrado ML, Pareja JA. Myofascial trigger points, neck mobility, and forward head posture in episodic tensiontype headache. Headache. 2007;47(5):662-72. doi: 10.1111/j.15264610.2006.00632.x. [PubMed: 17501847].

11. Cerezo-Tellez E, Torres-Lacomba M, Mayoral-Del Moral O, SanchezSanchez B, Dommerholt J, Gutierrez-Ortega C. Prevalence of myofascial pain syndrome in chronic non-specific neck pain: A populationbased cross-sectional descriptive study. Pain Med. 2016;17(12):2369-77. doi: 10.1093/pm/pnw114. [PubMed: 28025371]

12. Fleckenstein J, Zaps D, Ruger LJ, Lehmeyer L, Freiberg F, Lang PM, et al. Discrepancy between prevalence and perceived effectiveness of treatment methods in myofascial pain syndrome: results of a crosssectional, nationwide survey. BMC Musculoskelet Disord. 2010;11:32. doi: 10.1186/1471-2474-11-32. [PubMed: 20149248]. [PubMed Central: PMC2836281].

13. Lavelle ED, Lavelle W, Smith HS. Myofascial trigger points. Med Clin North Am. 2007;91(2):229-39. doi: 10.1016/j.mcna.2006.12.004 [PubMed: 17321283].

14. Testa M, Barbero M, Gherlone E. Trigger points: update of the clinical aspects. European Journal of Physical and Rehabilitation Medicine. 2003;39(1):37.

15. Park KD, Lee WY, Park MH, Ahn JK, Park Y. High- versus lowenergy extracorporeal shock-wave therapy for myofascial pain syndrome of upper trapezius: A prospective randomized single blinded pilot study. Medicine (Baltimore). 2018;97(28). e11432. doi: 10.1097/MD.0000000000011432. [PubMed: 29995794]. [PubMed Central: PMC6076058].

16. Müller-Ehrenberg H, Licht G. Diagnosis and therapy of myofascial pain syndrome with focused shock waves (ESWT). Med Orthop Tech. 2005;5:1-6.

17. Jeon JH, Jung YJ, Lee JY, Choi JS, Mun JH, Park WY, et al. The effect of extracorporeal shock wave therapy on myofascial pain syndrome. Ann Rehabil Med. 2012;36(5):665-74. doi: 10.5535/arm.2012.36.5.665. [PubMed: 23185731]. [PubMed Central: PMC3503942].

18. Xia P, Wang X, Lin Q, Cheng K, Li X. Effectiveness of ultrasound therapy for myofascial pain syndrome: a systematic review and meta-analysis. J Pain Res. 2017;10:545-55. doi: 10.2147/JPR.S131482. [PubMed: 28331357]. [PubMed Central: PMC5349701].

19. Machet L, Boucaud A. Phonophoresis: efficiency, mechanisms and skin tolerance. International Journal of Pharmaceutics. 2002;243(1-2):115. doi: 10.1016/s0378-5173(02)00299-5.

20. Unlu Z, Orguc S, Eskiizmir G, Aslan A, Tasci S. The role of phonophoresis in dyshpagia due to cervical osteophytes. Int J Gen Med. 2008;1:113. doi: 10.2147/ijgm.s3745. [PubMed: 20428400]. [PubMed Central: PMC2840538]

21. Takla MKN, Rezk-Allah SS. Immediate effects of simultaneous application of transcutaneous electrical nerve stimulation and ultrasound phonophoresis on active myofascial trigger points: A randomized controlled trial. Am J Phys Med Rehabil. 2018;97(5):332-8. doi: 10.1097/PHM.0000000000000876. [PubMed: 29206666].

22. Ay S, Dogan SK, Evcik D, Baser OC. Comparison the efficacy of phonophoresis and ultrasound therapy in myofascial pain syndrome. Rheumatol Int. 2011;31(9):1203-8. doi: 10.1007/s00296-010-14190. [PubMed: 20354859].

23. Ustun N, Arslan F, Mansuroglu A, Inanoglu D, Yagiz AE, Guler H, et al. Efficacy of EMLA cream phonophoresis comparison with ultrasound therapy on myofascial pain syndrome of the trapezius: a singleblind, randomized clinical study. Rheumatol Int. 2014;34(4):453-7. doi: 10.1007/s00296-013-2881-2. [PubMed: 24149990].

24. Sarrafzadeh J, Ahmadi A, Yassin M. The effects of pressure release, phonophoresis of hydrocortisone, and ultrasound on upper trapezius latent myofascial trigger point. Arch Phys Med Rehabil. 2012;93(1):72-7. doi: 10.1016/j.apmr.2011.08.001. [PubMed: 21982324].

25. Gür A. Comparison of the effectiveness of two different extracorporeal shock wave therapy regimens in the treatment of patients with myofascial pain syndrome. Arch Rheumatol. 2014;29(3):186-93. doi: 10.5606/ArchRheumatol.2014.3738.

26. Yoo JI, Oh MK, Chun SW, Lee SU, Lee CH. The effect of focused extracorporeal shock wave therapy on myofascial pain syndrome of trapezius: A systematic review and meta-analysis. Medicine (Baltimore). 2020;99(7). e19085. doi: 10.1097/MD.0000000000019085. [PubMed: 32049811]. [PubMed Central: PMC7035035].

27. Ramon S, Gleitz M, Hernandez L, Romero LD. Update on the efficacy of extracorporeal shockwave treatment for myofascial pain syndrome and fibromyalgia. Int J Surg. 2015;24(Pt B):201-6. doi: 10.1016/j.ijsu.2015.08.083. [PubMed: 26363497].

28. Haghighat S, Mashayekhi Asl M. Effects of extracorporeal shock wave therapy on pain in patients with chronic refractory coccydynia: A quasi-experimental study. Anesth Pain Med. 2016;6(4). e37428. doi: 10.5812/aapm.37428. [PubMed: 27843777]. [PubMed Central: PMC5098426].

29. Toghtamesh M, Tajali SB, Jalaei S. Comparing between the effects of dry needling and shock wave in the treatment of trapezius myofascial pain. J Mod Rehabil. 2020;14(4):225-32.

30. Akturk S, Kaya A, Cetintas D, Akgol G, Gulkesen A, Kal GA, et al. Comparision of the effectiveness of ESWT and ultrasound treatments in myofascial pain syndrome: randomized, sham-controlled study. J Phys Ther Sci. 2018;30(3):448-53. doi: 10.1589/jpts.30.448. [PubMed: 29581669]. [PubMed Central: PMC5857456]. 